\title{
Hepatic accumulation of Hedgehog-reactive progenitors increases with severity of fatty liver damage in mice
}

\author{
Susanne V Fleig ${ }^{1}$, Steve S Choi ${ }^{1}$, Liu Yang ${ }^{1}$, Youngmi Jung ${ }^{1}$, Alessia Omenetti ${ }^{1}$, Hendrika M VanDongen ${ }^{1}$, \\ Jiawen Huang ${ }^{1}$, Jason K Sicklick ${ }^{2}$ and Anna Mae Diehl ${ }^{1}$
}

Progenitors regenerate fatty livers but the mechanisms involved are uncertain. The Hedgehog pathway regulates mesendodermal progenitors and modulates mesenchymal-epithelial interactions during tissue remodeling. To determine if Hedgehog signaling increases in liver progenitors during fatty liver injury, we compared expression of Hedgehog ligands and target genes across a spectrum of injury. Leptin-deficient ob/ob mice with fatty livers and their healthy lean littermates were studied before and after exposure to the hepatotoxin, ethionine. At baseline, ob/ob mice had greater liver damage than controls. Ethionine induced liver injury in both ob/ob and lean mice, with greater injury occurring in ob/ob mice. After ethionine, the ob/ob mice developed liver atrophy and fibrosis. Liver injury increased hepatic accumulation of progenitors, including ductular cells that produced and responded to Hedgehog ligands. A dose-response relationship was demonstrated between liver injury and expansion of Hedgehog-responsive progenitors. In severely damaged, atrophic livers, nuclei in mature-appearing hepatocytes accumulated the Hedgehog-regulated mesenchymal transcription factor, Gli2 and lost expression of the liver epithelial transcription factor, hepatocyte nuclear factor 6 (HNF-6). Hepatic levels of collagen mRNA and pericellular collagen fibrils increased concomitantly. Hence, fatty liver injury increases Hedgehog activity in liver progenitors, and this might promote epithelial-mesenchymal transitions that result in liver fibrosis.

Laboratory Investigation (2007) 87, 1227-1239; doi:10.1038/labinvest.3700689; published online 22 October 2007

KEYWORDS: ethionine; epithelial-mesenchymal interaction; fatty liver; Hedgehog; progenitor

Hepatocyte apoptosis is increased in fatty livers, providing an ongoing stimulus for regeneration. ${ }^{1}$ Proliferation of mature hepatocytes is also inhibited in steatotic livers. Hence, their regeneration involves hepatic progenitor populations, ${ }^{2}$ and hepatic accumulation of progenitors increases with the severity of fatty liver damage. ${ }^{3,4}$ The mechanisms regulating this are poorly understood. In this study, we determined if fatty liver damage increases hepatic expression and activity of Hedgehog $(\mathrm{Hh})$ ligands, soluble factors that regulate mesendodermal progenitors.

In healthy adults, hepatic progenitors reside near Canals of Hering, vestiges of fetal liver ductal plates that house progenitors for both the hepatocyte and biliary epithelial cell compartments. ${ }^{2}$ Some progenitors are bi-potent, that is capable of differentiating along either epithelial lineage, while others are committed to a specific fate. ${ }^{5,6}$ Less certain is whether adult livers also retain more primitive multipotent progenitors, similar to mesendodermal cells that give rise to hematopoietic, endothelial, adipocytic, smooth muscle-like, and epithelial cell types during embryogenesis. ${ }^{7,8}$

Development of many tissues, including the liver, requires migration of the mesendoderm during gastrulation. ${ }^{9}$ Recently, mesendodermal-like cells expressing the epithelial marker, EpCAM, were isolated from human fetal livers. ${ }^{10}$ When transplanted into adult mice with liver injury, these $\operatorname{EpCAM}(+)$ cells integrated into hepatic parenchyma and differentiated into functioning hepatocytes. Gene expression analysis of cultured EpCAM $(+)$ cells undergoing hepatocyte differentiation demonstrated that their initially strong expression of mesenchymal markers declined as hepatocyte gene expression was acquired, leading the authors to suggest that fetal liver mesendodermal cells are capable of mesenchymal-epithelial transitions.

Human fetal liver EpCAM $(+)$ cells produce and respond to Hedgehog (Hh) ligands. ${ }^{11}$ Hh ligands are soluble factors that interact with the plasma membrane-spanning receptor,

\footnotetext{
${ }^{1}$ Division of Gastroenterology, Department of Medicine, Duke University, Durham, NC, USA and 2 Department of Surgery, Johns Hopkins University, Baltimore, MD, USA Correspondence: Dr AM Diehl, MD, Division of Gastroenterology, Department of Medicine, Duke University Medical Center, GSRB No. 1595 LaSalle Street, Suite 1073 , Box 3256, Durham, NC 27710, USA. E-mail: annamae.diehl@duke.edu
} 
Patched (Ptc), to de-repress the downstream intracellular signaling intermediate, Smoothened (Smo). Smo-initiated signals, in turn, activate Gli-family transcription factors to induce expression of Hh-responsive genes that regulate target cell proliferation, viability and differentiation. ${ }^{12}$ During embryogenesis, Hh ligands function as morphogens by modulating both mesendodermal fate and mesenchymalepithelial transitions. ${ }^{13-15}$ Many adult tissues also contain mesendodermal-like cells and mesenchymal-epithelial interactions mediate adult wound healing. ${ }^{16,17}$ Consistent with these observations is growing evidence that $\mathrm{Hh}$ signaling regulates remodeling of various adult tissues, including the nervous system, skin, heart, lung, and gastrointestinal tract. ${ }^{18-21}$ Excessive Hh activity has also been noted in cancers arising in these tissues. ${ }^{22-24}$

Hh signaling has been reported in some hepatocellular carcinomas and cholangiocarcinomas, ${ }^{22,23}$ although mature liver epithelial cells in healthy adult livers lack obvious Hh pathway activity. ${ }^{11}$ Recently, increased hepatic expression of Hh ligands and target genes was demonstrated in adult rodents following bile duct ligation ${ }^{25}$ and in humans with primary biliary cirrhosis. ${ }^{26}$ In both rodents and humans, cholestatic liver damage triggered expression of $\mathrm{Hh}$ ligands, the Hh receptor, Ptc, and Hh-regulated transcription factors, such as Gli-2, in bile ductular cells and peri-ductular stromal cells, some of which coexpressed OV-6, a marker for bipotent liver epithelial progenitors (ie, oval cells), ${ }^{6}$ or $\alpha$-smooth muscle actin, a marker for liver myofibroblasts. $^{27,28}$ Coculturing liver myofibroblastic cells with immature bile ductular cells increased Hh pathway activity and enhanced the growth of both cell types. Both effects were inhibited by Hh-neutralizing antibodies, suggesting that soluble Hh ligands provide viability signals for subpopulations of myofibroblastic and bile ductular cells in adult livers.

The present study evaluates the hypothesis that injury to mature hepatocytes also triggers expansion of Hh-responsive progenitor populations. To evaluate this concept, we characterized progenitor populations and Hh pathway activity in healthy, lean adult mice and in obese, ob/ob mice with genetic leptin deficiency and fatty livers. ${ }^{4}$ Steatosis is one of the most common forms of hepatic pathology in humans, ${ }^{29}$ and ob/ob mice, like many obese people, spontaneously acquire nonalcoholic steatohepatitis (NASH), but do not develop liver atrophy or significant liver fibrosis despite having chronic liver injury. ${ }^{30,31}$ Adult ob/ob mice and their healthy littermates were studied at baseline and after exposure to ethionine, a toxin for mature hepatocytes that promotes the rapid outgrowth of hepatic progenitor populations. We demonstrate a dose-response relationship between mature hepatocyte injury and expansion of liver progenitor populations that produce and respond to $\mathrm{Hh}$ ligands, and suggest that $\mathrm{Hh}$ signaling may modulate mesenchymal-epithelial transitions during remodeling of damaged livers.

\section{MATERIALS AND METHODS}

\section{Animal Studies}

Eight-week-old, male C57BL6 ob/ob mice $(n=25)$ and lean littermates $(n=15)$ (Jackson Laboratory, Bar Harbor, ME, USA) were fed normal chow. To induce oval cell proliferation, DL-ethionine (Alfa Aesar, Lancaster, England) at concentrations of $0.15 \%$ and $0.075 \%$ was added to the drinking water of ob/ob and lean mice. Control mice (5 per group) drank untreated water. After 1 week, animals were killed and the liver and blood samples were harvested. The studies were approved by the Duke University Animal Committee and fulfilled NIH guidelines.

\section{Immunohistochemistry}

Formalin-fixed, paraffin-embedded livers were prepared for immunohistochemistry as described. ${ }^{25,26}$ Sections were incubated with primary antibodies, A6 (gift from Dr Valerina Factor, NIH, Bethesda, MD, USA), CD56/NCAM (Vector Lab, Burlingame, CA, USA), CK19 (DAKO Corporation, Carpinteria, CA, USA), Gli2 (Abcam, Cambridge, MA, USA), HNF-6 (Santa Cruz Biotechnology, Santa Cruz, CA, USA), Ihh (Santa Cruz Biotechnology) and $\alpha$-smooth muscle actin ( $\alpha \mathrm{SMA})(\mathrm{DAKO})$ at $4^{\circ} \mathrm{C}$ overnight. Other sections were similarly exposed to nonimmune sera. Secondary antibodies were goat anti-rat IgG-HRP (Santa Cruz Biotechnology) for A6, polymer-HRP anti-rabbit (DAKO) for HNF-6, Ihh and Gli2 and polymer-HRP anti-mouse (DAKO) for CD56, CK19 and $\alpha$ SMA. Antigens were demonstrated by diaminobenzidine (DAB) (DAKO). Omitting primary antibodies eliminated staining, demonstrating specificity. After excluding the major bile duct in each portal tract (PT) from consideration, cells staining positively for CK19, HNF-6, Gli2, Ihh, or A6 in $10 \mathrm{PT} /$ slide were counted at $\times 40$ magnification. Hepatocyte nuclear staining for HNF-6 was quantified in 10 fields/slide; $\operatorname{HNF}(+)$ hepatocytes/field were divided by the total number of hepatocytes/field to control for the fact that ob/ob hepatocytes are fatty and therefore larger, reducing the number of hepatocytes/field.

\section{Two-Step Real-Time Reverse Transcription-Polymerase Chain Reaction}

Total liver RNA was extracted using TRIzol (Invitrogen, Carlsbad, CA, USA). After RNase-free DNase I treatment (Qiagen, Valencia, CA, USA), RNA was reverse transcribed to cDNA templates using random primer and Superscript RNase H-reverse transcriptase (Invitrogen) and amplified. For semi-quantitative RT-PCR, $1.5 \%$ of the first-strand reaction was amplified using iQ-SYBR Green Supermix (BioRad, Hercules, CA, USA), an iCycler iQ Real-Time Detection System, and specific oligonucleotide primers for target sequences, as well as the $18 \mathrm{~S}$ housekeeping gene (Table 1). Threshold cycles $\left(C_{t}\right)$ were automatically calculated by the iCycler iQ Real-Time Detection System. Target gene levels in the cells are presented as a ratio to levels detected in the corresponding control cells according to the $\Delta \Delta C_{\mathrm{t}}$ method. 
Table 1 Sequences of PCR primers used in experiments

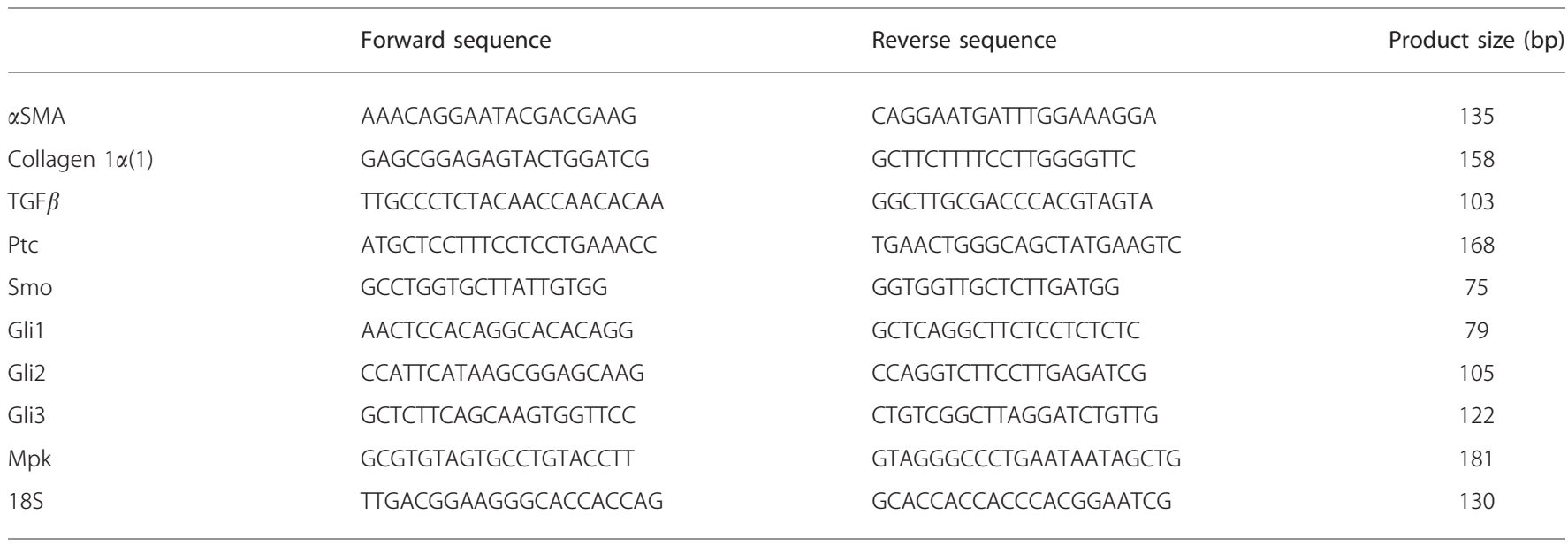

Amplicon products were then separated by electrophoresis on a $2.0 \%$ agarose gel buffered with $0.5 \times \mathrm{TBE}$.

\section{Morphometry}

Collagen staining of formalin-fixed liver sections with picrosirus red was assessed by morphometry with MetaView software (Universal Imaging, Downington, PA, USA). Twelve randomly selected $20 \times$ fields/section were evaluated for each mouse. $^{32}$

\section{Terminal Deoxynucleotidyl Transferase-Mediated dUDP Nick-End Labeling Assay}

Terminal deoxynucleotidyl transferase-mediated dUDP nick-end labeling (TUNEL) assay was performed using a kit (Boehringer Mannheim, Mannheim, Germany) as described previously. $^{33}$

\section{Hydroxyproline Assay}

Hepatic hydroxyproline content was quantified colorimetrically in flash-frozen liver samples. ${ }^{34}$ Concentrations were calculated from a standard curve prepared with highpurity hydroxyproline (Sigma, St Louis, MO, USA) and expressed as mg hydroxyproline/g liver.

\section{Statistical Analysis}

Results are expressed as means \pm s.e.m., unless indicated otherwise. Comparisons between groups were performed using the Student's $t$-test. Significance was accepted at the $5 \%$ level.

\section{RESULTS}

\section{ob/ob Mice Spontaneously Develop NASH}

The ob/ob mice were obese and had hepatomegaly (Table 2). Their livers were also more fatty (Figure $1 \mathrm{a}$ and $\mathrm{b}$ ). Inflammatory cell infiltrates were rare and fibrosis was not observed, although hepatocyte ballooning was frequent (Figure 1b, inset). Serum markers of liver injury (Table 2) and collagen mRNA levels (Figure 1c) were also significantly higher in ob/ob mice, consistent with reports that they spontaneously develop NASH. ${ }^{30}$

\section{Hepatic Accumulation of Bile Ductular Cells in ob/ob Mice with NASH}

To assess regenerative responses to fatty liver damage, we compared progenitor populations in ob/ob and control mice. Portal tracts (PT) in fatty livers harbored more progenitor cells, including increased numbers of cells expressing A6, a marker for bipotent hepatic epithelial progenitors (ie, oval cells) and bile ductular cells (Figure $2 \mathrm{a}-\mathrm{c}$ ), NCAM, a marker for oval cells and immature bile ductular cells (Figure 2d-f), and CK 19, a marker of immature and mature bile ductular cells (Figure $2 \mathrm{~g}-\mathrm{i}$ ). ${ }^{6}$ Thus, bile ductular cells of varying degrees of maturation accumulated in livers with NASH. A similar ductular response has been noted in other types of chronic liver injury, and is considered to be involved in liver repair in humans, as well as rodents. ${ }^{3}$

\section{Increased Ihh Ligand and Hh Pathway Activity in ob/ob Mice with NASH}

We next compared mRNA and protein expression of $\mathrm{Hh}$ ligands in lean and ob/ob livers. Although whole liver mRNA levels of sonic Hedgehog $(s h h)$ and Indian Hedgehog (ihh) were not increased in ob/ob livers (data not shown), immunohistochemistry demonstrated significantly increased peri-portal accumulation of Ihh $(+)$ cells (Figure 3A-C). In both control (Figure $3 \mathrm{Aa}$ and $\mathrm{Ba}$ ) and ob/ob mice (Figure $3 \mathrm{Ab}$ and $\mathrm{Bb})$, most of the Ihh-expressing cells were bile ductular cells. Some stromal cells in and around PT of ob/ob livers also expressed Ihh. To determine if expression of $\mathrm{Hh}$ ligand correlated with increased $\mathrm{Hh}$ pathway activity, we evaluated mRNA expression of Hh-regulated transcription factors. Genes encoding Gli1 and Gli2 are induced by $\mathrm{Hh}$ signaling. Thus, expression of these factors identifies cells with Hh pathway activity. ${ }^{13}$ Expression of Gli1, Gli2 and Gli3 
Table 2 Comparison of WT and ob/ob mice-ob/ob mice spontaneously develop NASH

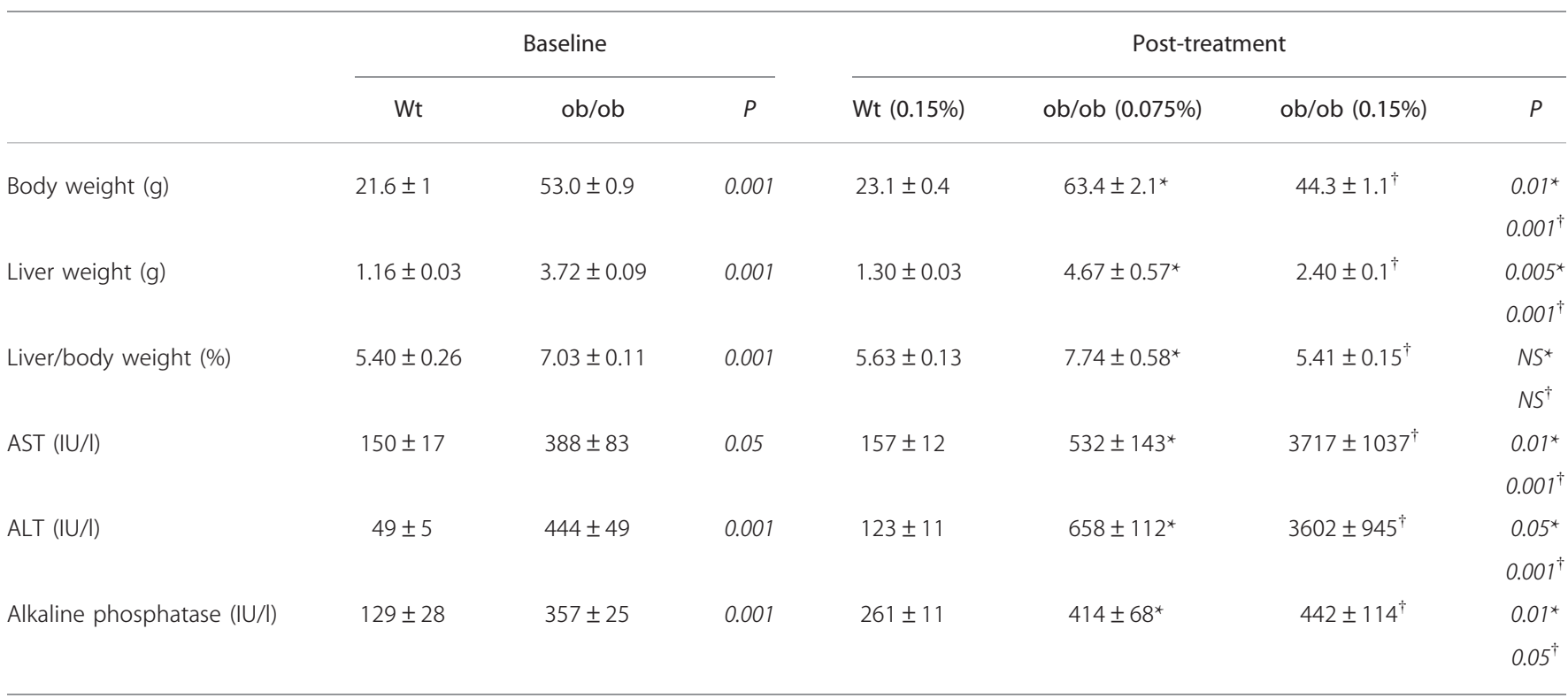

ob/ob mice were obese and had hepatomegaly. Serum markers of liver injury were also significantly higher in ob/ob mice, consistent with the development of $\mathrm{NASH} .{ }^{*}=v s \mathrm{Wt}(0.15 \%) ;{ }^{\dagger}=v s \mathrm{Wt}(0.15 \%)$.
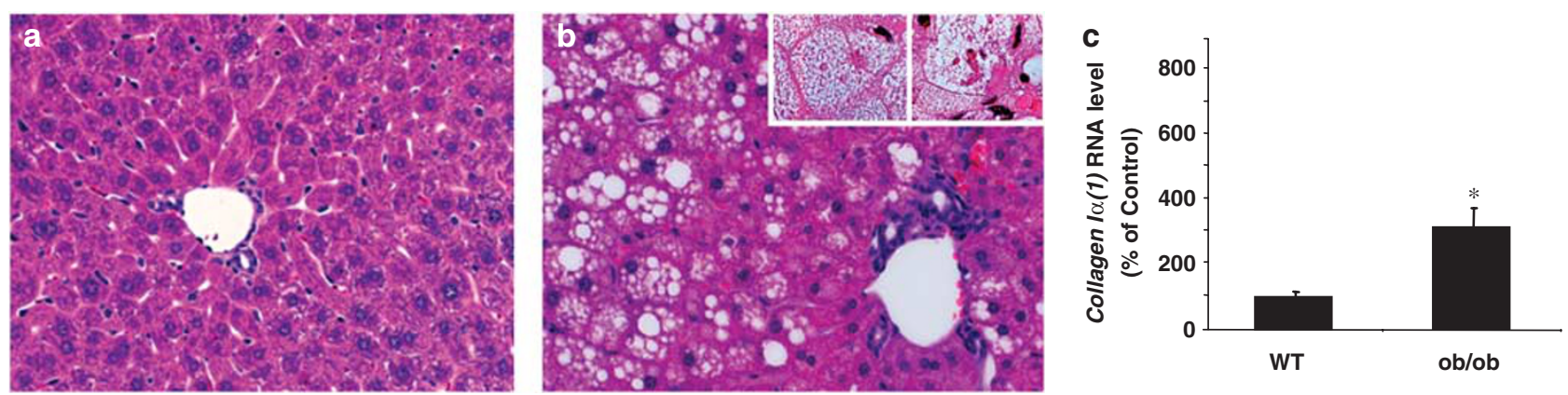

Figure $1 \mathrm{NASH}$ and increased collagen gene expression in ob/ob mice. H\&E from representative lean (WT) (a) and ob/ob mice (b) at $\times 400$ magnification. QRT-PCR analysis of collagen $1 \alpha(\mathrm{I})$ in whole liver tissue from WT $(n=5)$ and ob/ob $(n=5)$ mice. Data are displayed as mean \pm s.e.m. $\left({ }^{*} P<0.05 \%\right.$ vs WT).

mRNA was similar in ob/ob and lean livers (data not shown), so immunohistochemistry was performed to localize Gliexpressing cells. Either absent or faint, diffuse non-specific staining occurred with antibodies for Gli1 and Gli3, but Gli2 proved useful for immunohistochemistry. In normal livers, sinusoidal cells throughout the lobule, and some bile ductular cells in PT, stained positive for Gli2. Mature hepatocytes did not express this protein (Figure 3Ad). In ob/ob livers, the overall number of Gli2 $(+)$ cells was only somewhat greater than in control livers (Figure 3Cb). However, the pattern of staining was very different (Figure $3 \mathrm{Ac}-\mathrm{d}$ ). ob/ob livers harbored few Gli2 $(+)$ sinusoidal cells, but many more Gli2positive cells in PT. Most of these Gli2-positive cells were bile ductular cells, although increased numbers of small Gli2expressing stromal cells were also noted in peri-portal regions. Hence, populations of bile ductular cells and portal stromal cells that produce and respond to Hh ligands expanded in livers with NASH.

\section{ob/ob Mice are Particularly Vulnerable to Ethionine Hepatotoxicity}

To further address the role of Hh signaling in the progenitor response to parenchymal liver injury, we treated mice with ethionine. Ethionine promotes hepatic necrosis by eliciting oxidative stress and ATP depletion in hepatocytes. ${ }^{35}$ Similar mechanisms underlie the pathogenesis of NASH. ${ }^{36}$ Fatty livers are particularly vulnerable to ethionine-induced hepatotoxicity, rapidly accumulating hepatic progenitors when exposed to this drug. ${ }^{3,37}$ Ethionine was administered in drinking water for 1 week, ${ }^{37}$ and water consumption in the two groups of mice was similar. Thus, ob/ob mice and controls ingested similar total doses of ethionine at full-dose 

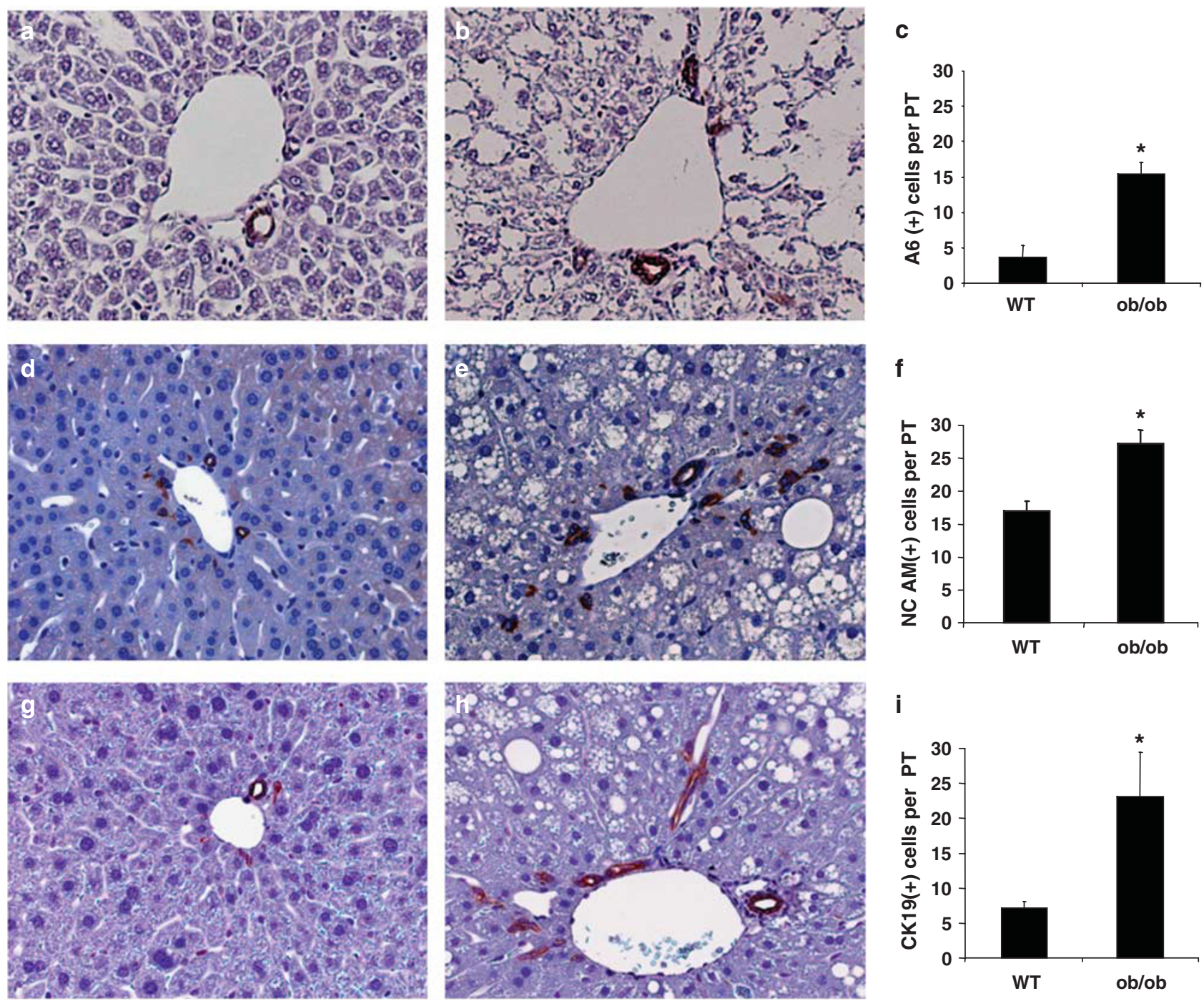

Figure 2 Increased liver progenitor cell populations in ob/ob mice. A6 (a, b), NCAM (d, e) and CK19 (g, h) immunohistochemistry in representative WT $(\mathbf{a}, \mathbf{d}$ and $\mathbf{g})$ and ob/ob (b, e and $\mathbf{h})$ mice at $\times 400$ magnification. Positive cells for these markers were counted in 10 portal tracts (PT)/section in WT ( $n=5$ ) and ob/ob $(n=5)$ mice $\left({ }^{*}<<0.05\right)$.

$(0.15 \%)$ treatment, although the ethionine dose/g body weight was significantly lower in morbidly obese, ob/ob mice than lean controls. Despite this, ob/ob mice suffered much worse ethionine toxicity.

No lean mice died or lost significant body weight during ethionine treatment, whereas only $3 / 10 \mathrm{ob} / \mathrm{ob}$ mice treated with maximal dose $(0.15 \%)$ ethionine survived $(P<0.001)$ with all survivors demonstrating significant weight loss (Table 2 ). At the reduced dose of $0.075 \%$ ethionine, all ob/ob mice survived. Ethionine caused liver atrophy in surviving ob/ob mice treated with $0.15 \%$ ethionine but did not perturb liverto-body weight ratios in lean mice (Table 2). Although lean mice did not become overtly ill during ethionine treatment, their serum values of aminotransferases and alkaline phosphatase doubled, suggesting mild liver injury. AST and ALT values also increased slightly in ob/ob mice treated with
$0.075 \%$ ethionine. In contrast, aminotransferase values increased by almost 10 -fold in surviving ob/ob mice treated with full-dose $(0.15 \%)$ ethionine. Consequently, the ob/ob group had significantly higher serum aminotransferase values than controls at the end of treatment (Table 2). Serum values of the bile ductular enzyme, alkaline phosphatase, were only also higher in ob/ob mice than in lean controls following ethionine treatment (Table 2). Heightened parenchymal liver injury in ob/ob mice was confirmed by TUNEL staining (Figure 4).

\section{Ethionine Alters Progenitor Populations in Lean and ob/ob Livers}

Ethionine elicited PT expansion in both groups, but this response was greater in ob/ob than lean mice (Figure $5 \mathrm{a}$ and $\mathrm{b}$ ). Although ethionine generally increased PT accumulation of 
A


C

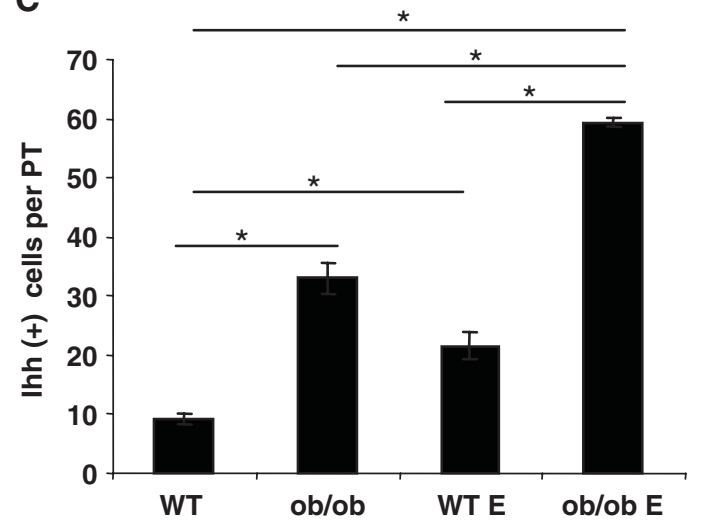

B


Figure 3 Increased Ihh ligand expression and Hh activity in ob/ob mice. Ihh immunohistochemistry in representative WT (a) and ob/ob (b) mice from untreated (A) and post-ethionine (B) groups. Gli2 immunohistochemistry in representative WT (c) and ob/ob (d) mice from untreated (WT and ob/ob) (A) and post-0.15\% ethionine (WT E $0.15 \%$ and ob/ob E 0.15\%) groups (B). Magnification $\times 400$. Positive cells for these markers were counted in 10 PT/section from mice in the various treatment groups (WT control, $n=5$, WT E $0.15 \%, n=10$; ob/ob control, $n=5$, ob/ob E $0.15 \%, n=3$ ) and graphed (C) as mean \pm s.d. $\left({ }^{*}<0.05\right)$.
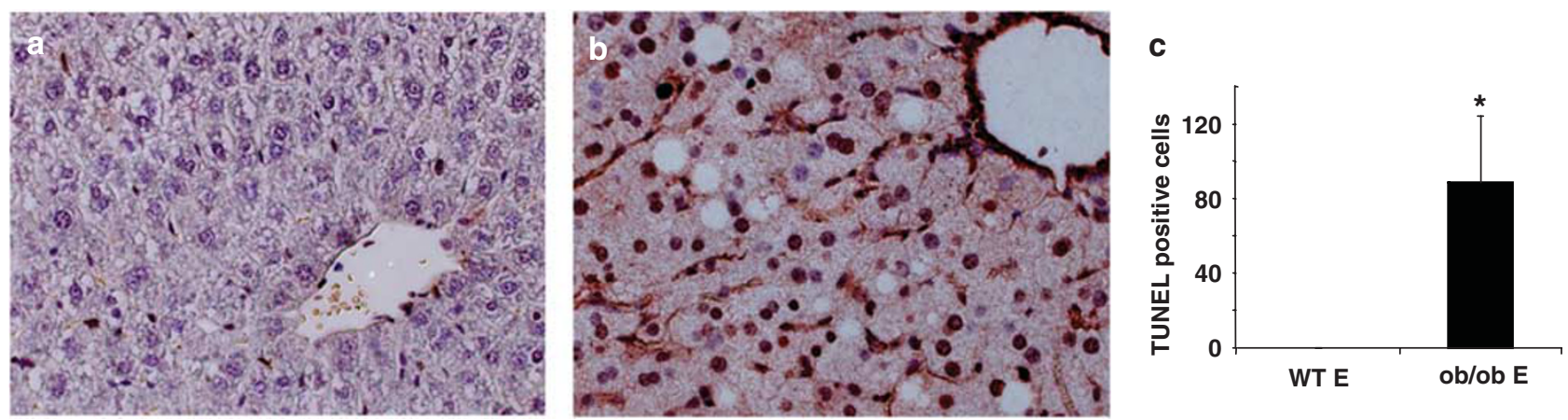

Figure 4 Vulnerability to ethionine hepatotoxicity in ob/ob mice. TUNEL assay was performed on formalin-fixed, paraffin-embedded liver sections from ethionine-treated WT (WT E 0.15\%) (a) and ob/ob (ob/ob E 0.15\%) (b) mice (WT E 0.15\%, $n=10$; ob/ob E 0.15\%, $n=3$ ). TUNEL-positive cells were quantified (c) and shown as mean \pm s.d. $\times 400$ magnification $\left({ }^{*} P<0.05\right.$ vs WT E $\left.0.15 \%\right)$.

$\mathrm{A} 6(+)$ progenitors, the net number of $\mathrm{A} 6(+)$ cells/PT was greater in ob/ob than lean mice (Figure $5 \mathrm{c}-\mathrm{e}$ ). The characteristics of the progenitor populations that accumu- lated post-ethionine also differed in the two groups (Figure $5 \mathrm{f}-\mathrm{k})$. In lean mice, ethionine induced comparable, two- to three-fold increases in A6 $(+)$ cells and CK-19(+) cells. In 



e
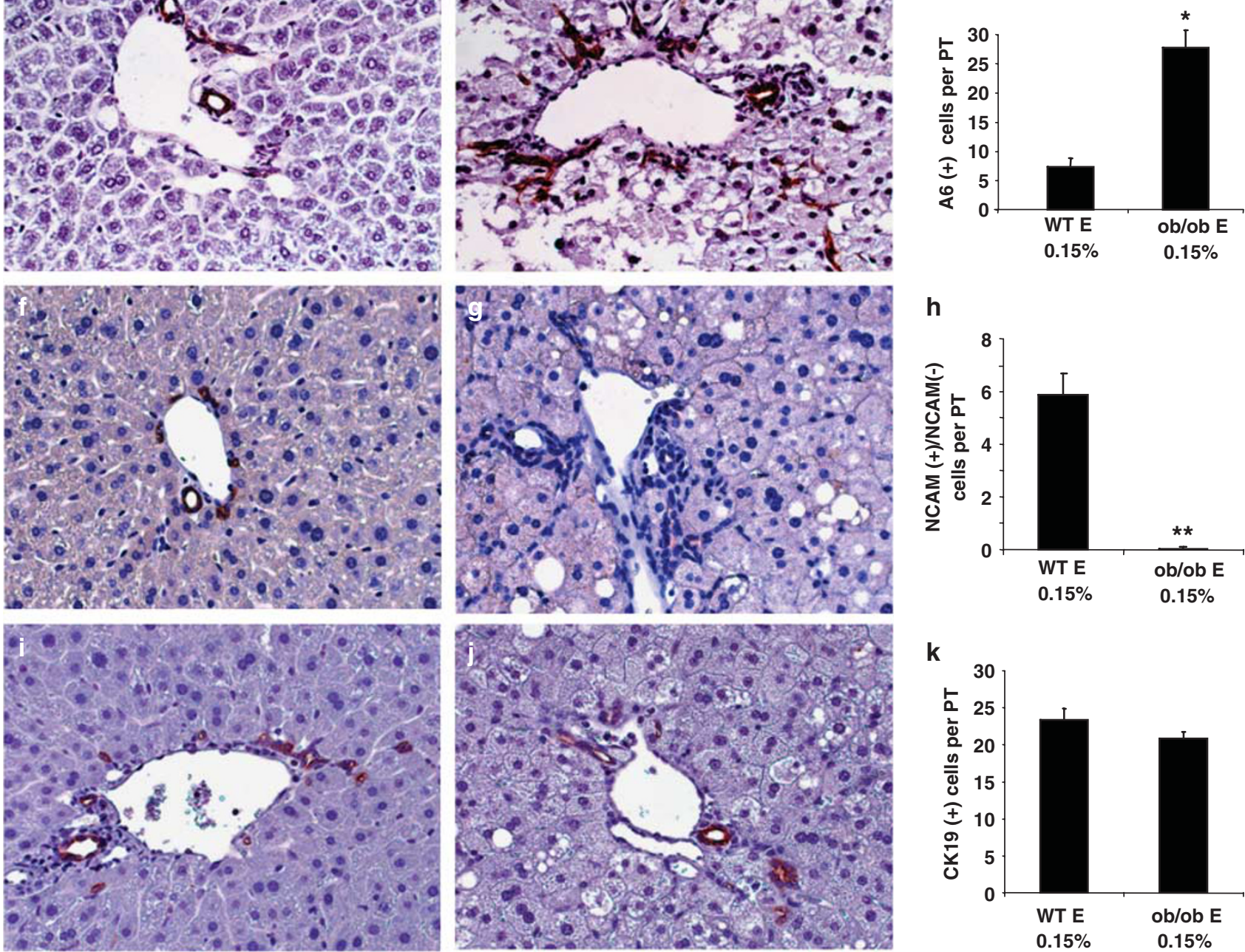

Figure 5 Differential progenitor population expansion in lean and ob/ob livers after ethionine. ob/ob mice and lean (WT) littermates were exposed to ethionine at doses of $0.075 \%$ (ob/ob E $0.075 \%$ ) and $0.15 \%$ (ob/ob E $0.15 \%$ ) for 1 week ( $n=10 /$ group). H\&E from representative WT E $0.15 \%$ (a) and ob/ob E $0.15 \%$ (b) mice. A6 (c, d), NCAM (f, g) and CK19 (i, j) immunohistochemistry in representative WT and ob/ob mice at $\times 400$ magnification. Positive cells for these markers were counted in $10 \mathrm{PT} /$ section in mice that received the highest ethionine dose (WT E $0.15 \%, n=10 ;$ ob/ob E $0.15 \%, n=3$ ) and graphed as mean \pm s.d. $\left({ }^{*} P<0.05 \%\right.$ vs WT E $\left.0.15 \% ;{ }^{* * P}<0.005\right)$.

ethionine-treated ob/ob mice, the numbers of CK-19 $(+)$ cells remained constant, despite accumulation of $\mathrm{A} 6(+)$ cells. Hence, ob/ob and lean mice ultimately exhibited similar numbers of $\mathrm{CK} 19(+)$ cells/PT after ethionine exposure.
Also, although ethionine decreased $\operatorname{NCAM}(+)$ cells in both groups, lean mice retained more $\operatorname{NCAM}(+)$ cells than $\mathrm{ob} / \mathrm{ob}$ mice, in which $\operatorname{NCAM}(+)$ cells became virtually undetectable. 


\section{Hh Pathway Activation Parallels Hepatocyte Injury and Progenitor Response}

To determine how ethionine-induced changes in liver progenitor populations related to changes in Hh pathway activity, total liver RNA was isolated and analyzed by Q-RTPCR. Although whole liver expression of shh and ith were similar in WT and 0.075 and $0.15 \%$ ethionine-treated ob/ob groups (data not shown), ob/ob mice exhibited greater expression of the Hh receptor (Ptc) (Figure 6a), co-receptor (Smo) (Figure 6b), and several Hh-target genes (Gli1, Gli2 and Gli3) than lean mice (Figure $6 \mathrm{c}-\mathrm{e}$ ). Within the ob/ob group, Hh pathway activity increased in parallel with the severity of ethionine-induced liver damage. Similarly, ethionine treatment of ob/ob mice resulted in marked dose-related increases in the expression of muscle pyruvate kinase (mpk), a marker of liver progenitors (eg, oval cells) ${ }^{38,39}$ (Figure 6f).

Since RNA analysis had proven to be insensitive for detecting changes in Hh expression/activity that occurred in subpopulations of liver cells, Ihh and Gli2 expressions were assessed by immunohistochemistry. Ethionine increased hepatic accumulation of Ihh-producing cells, as well as $\mathrm{Hh}$ responsive cells that expressed Gli2 (Figure 3). The greatest numbers of cells staining positive for Ihh and Gli2 were noted in $0.15 \%$ ethionine-treated $\mathrm{ob} / \mathrm{ob}$ mice. In ob/ob mice, fulldose ethionine also elicited accumulation of Gli2 in the nuclei of many mature-appearing hepatocytes. This was intriguing because Gli2 is a transcriptional regulator for mesenchymal cells, and ob/ob mice that survived acute-on-chronic liver injury exhibited liver fibrosis and atrophy (Table 2).

\section{Decreased Expression of Liver Cell Transcription Factor, Increased Hepatocyte Expression of Gli2 and Altered Tissue-Specific Gene Expression in ob/ob Livers}

These findings prompted us to evaluate expression of HNF-6, a transcriptional regulator of liver epithelial cells. ${ }^{40}$ Basal expression of HNF-6 differed in ob/ob and control livers (Figure $7 \mathrm{a}-\mathrm{c}$ ). Although bile ductular cells demonstrated strong nuclear accumulation of HNF-6 in both groups, hepatocyte nuclei stained more strongly for HNF-6 in control livers. The proportion of hepatocytes expressing detectable HNF-6 was also reduced in ob/ob livers. Nevertheless, ob/ob livers maintained many critical, tissue-specific functions. Serum bilirubin levels and prothrombin times were similar in $\mathrm{ob} / \mathrm{ob}$ mice and controls and serum albumin levels were actually higher in the ob/ob group (data not shown).
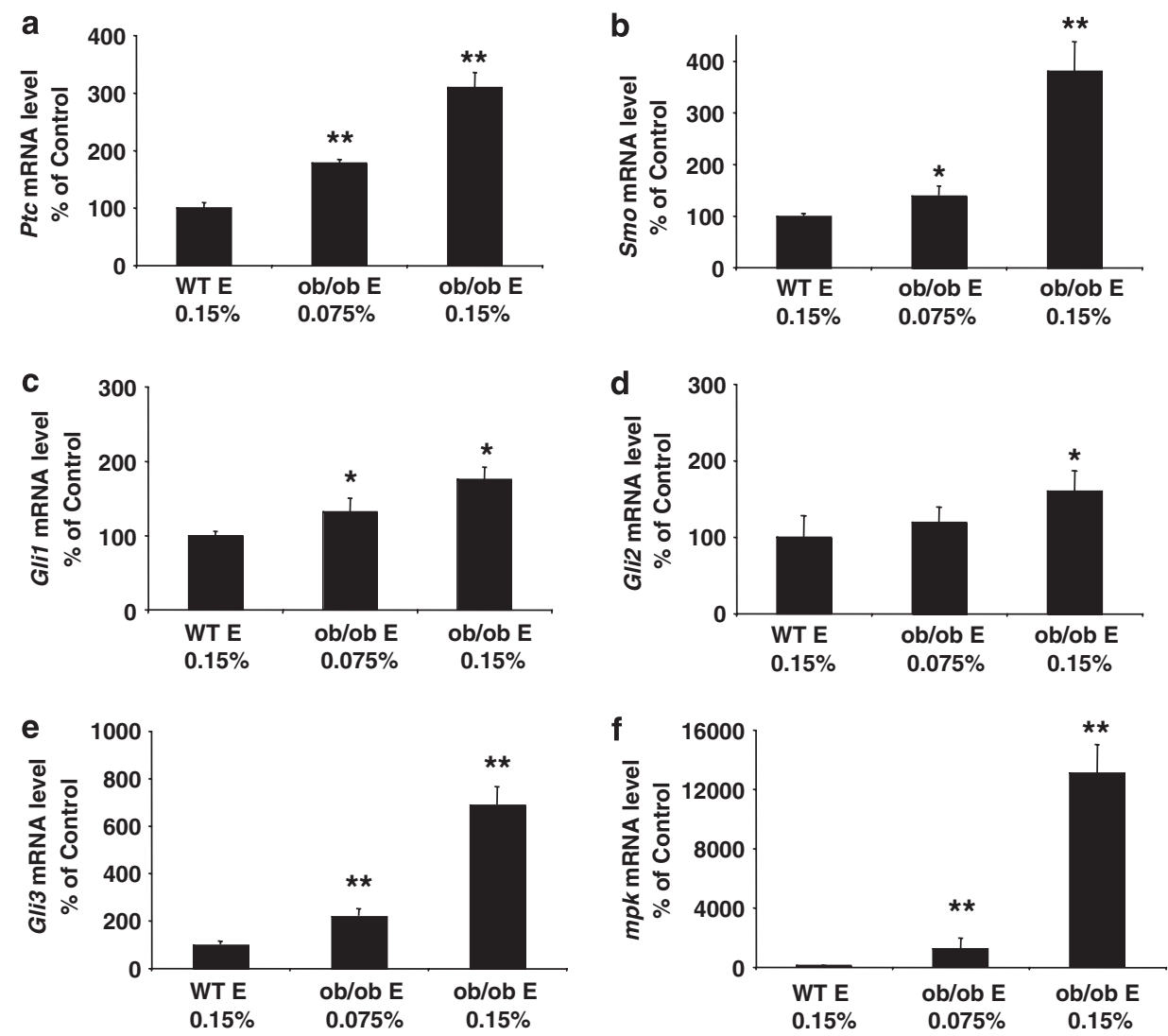

Figure 6 Increased hedgehog pathway expression in ethionine treated ob/ob mice. QRT-PCR analysis of Ptc (a), Smo (b), Gli1 (c), Gli2 (d), Gli3 (e), and mpk (f) in whole liver tissue from lean (WT E $0.15 \%, n=10$ ), and ob/ob (ob/ob E 0.075\%, $n=10$; ob/ob E 0.15\%, $n=3$ ) mice after ethionine treatment. Mean \pm s.e.m. $\left({ }^{*} P<0.05 \%\right.$ vs WT E $0.15 \% ;{ }^{* * P}<0.001 \%$ vs WT E $\left.0.15 \%\right)$. 



Figure 7 Differential HNF-6 expression in ob/ob and control livers. HNF-6 immunohistochemistry in representative WT (a, d) and ob/ob (b, e) mice from untreated $(\mathbf{a}, \mathbf{b})$ and ethionine treated $(\mathbf{d}, \mathbf{e})$ groups at $\times 400$ magnification. An asterisk indicates one of HNF- $6(+)$ hepatocytes in ethionine-treated WT mouse. HNF-6( + ) cells were counted in 10 PT/section from mice that received the highest dose of ethionine $(\mathrm{WT} E \mathrm{E} 0.15 \%, n=10 ; \mathrm{ob} / \mathrm{ob} \mathrm{E} 0.15 \%, n=3)$ expressed as \% of HNF-6(+) nuclei/total hepatocytes, and graphed as mean \pm s.d. (c, f). QRT-PCR analysis of HNF-6 (g) in whole liver tissue from lean (WT E $0.15 \%, n=10)$ and ob/ob (ob/ob E $0.075 \%, n=10 ; \mathrm{ob} / \mathrm{ob} \mathrm{E} 0.15 \%, n=3)$ mice after ethionine treatment. Results are graphed as mean \pm s.e.m. $\left({ }^{*} P<0.05 \%\right.$ vs WT E $0.15 \% ;{ }^{* \star P}<0.001 \%$ vs WT E $\left.0.15 \%\right)$.

In lean mice, ethionine had no effect on hepatocyte nuclear accumulation of HNF-6. However, it abolished the low basal expression of HNF-6 in ob/ob hepatocytes (Figure 7df). Consistent with this finding, treatment of ob/ob mice with ethionine suppressed HNF-6 mRNA expression (Figure 7g). These effects were cell-specific, because bile ductular and stromal cell staining for HNF-6 was not affected by ethionine. Ethionine also significantly increased nuclear accumulation of Gli2 in ob/ob hepatocytes (Figure 3). Hepatocytes in untreated $\mathrm{ob} / \mathrm{ob}$ mice, healthy lean mice and ethionine-treated lean mice did not express Gli2.

Induction of Gli2 in ob/ob hepatocytes correlated with increased pericellular fibrosis in ob/ob livers (Figure 8). Gli activity promotes epithelial-mesenchymal transitions. ${ }^{13,18,41,42}$
Therefore, we compared fibrosis markers in ethionine-treated lean and ob/ob mice. Although others have reported reduced fibrotic responses in ob/ob mice, ${ }^{43,44}$ we found that ob/ob mice expressed higher mRNA levels of collagen $1 \alpha I$ (coll $\alpha I)$ (Figure 8a), asma (Figure 8b), and transforming growth factor- $\beta$ (TGF- $\beta$ ) (Figure 8c) than lean mice after ethionine treatment. As noted for Hh pathway gene expression, expression of these fibrogenic markers increased in a dose-dependent manner and were greatest in the subset of $\mathrm{ob} / \mathrm{ob}$ mice that received the higher ethionine dose that caused the most liver injury. Immunohistochemistry confirmed that livers from ob/ob mice treated with $0.15 \%$ ethionine contained more Sirius-red-stained fibrils than the livers from lean controls after ethionine exposure (Figure 8d-f). During this 
a

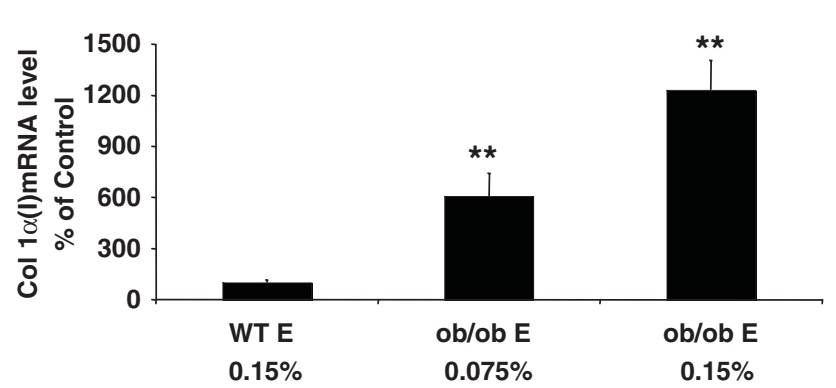

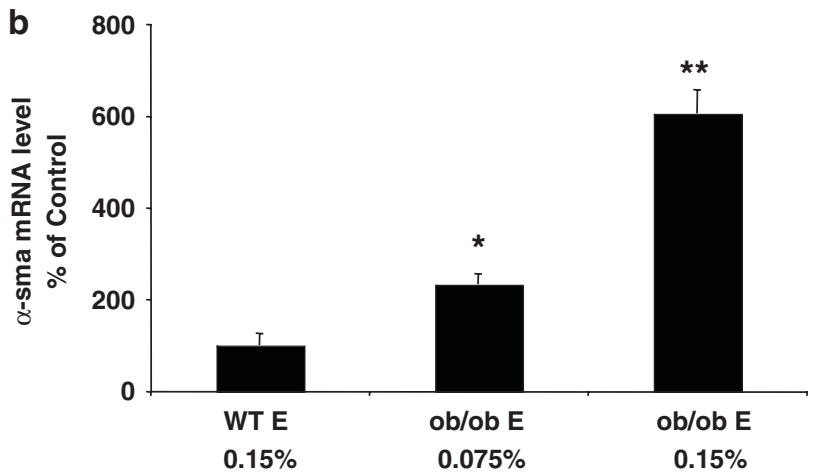
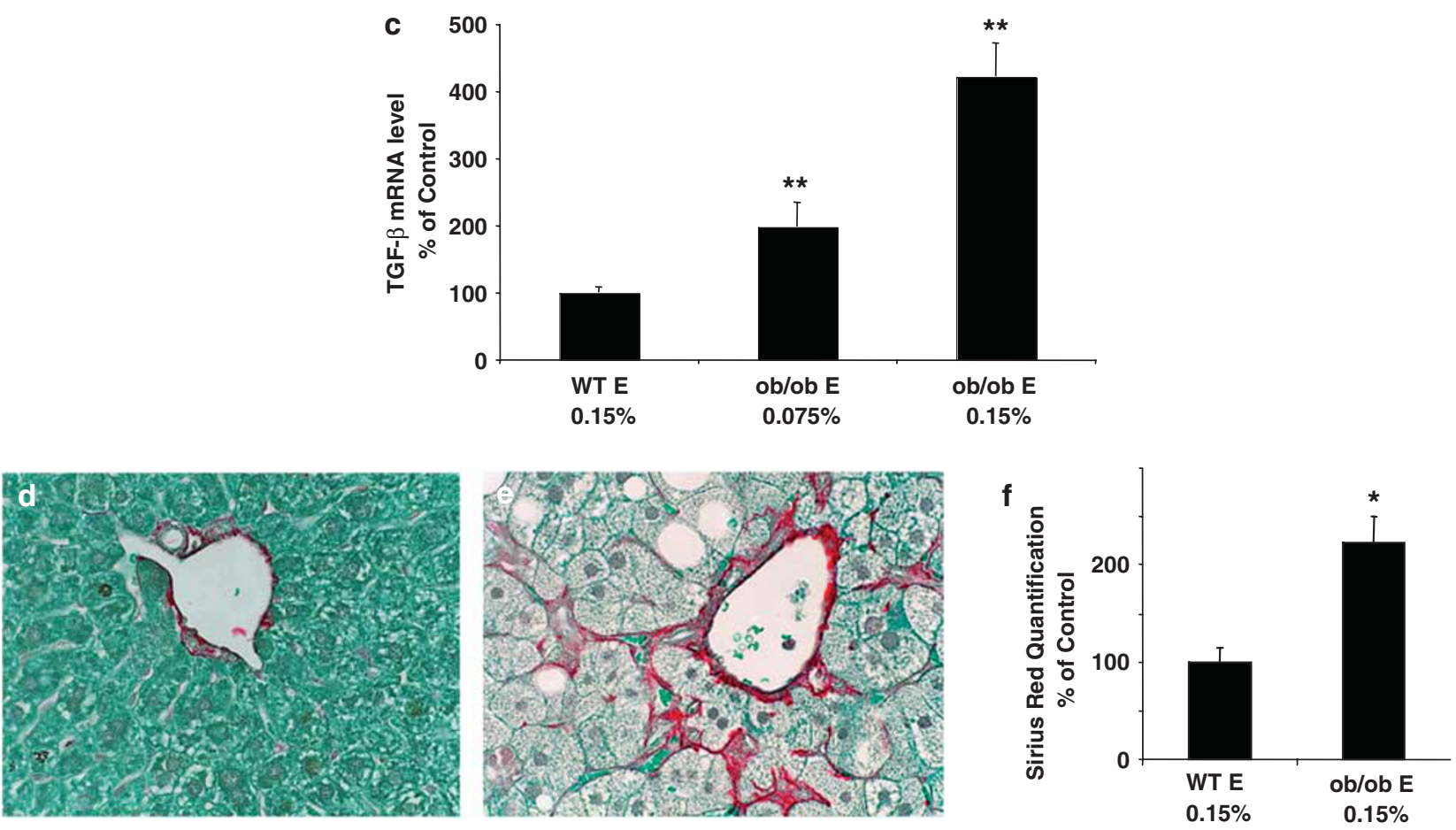

Figure 8 Increased fibrosis markers in ethionine-treated ob/ob mice. Ob/ob mice $(n=20)$ and lean wild-type littermates (WT) ( $n=10)$ were exposed to ethionine for 1 week. QRT-PCR analysis (a-c) of collagen $1 \alpha(\mathrm{I})(\mathbf{a}), \alpha \mathrm{SMA}(\mathbf{b})$, and TGF- $\beta$ (c) in whole liver tissue from all surviving lean (WT E 0.15\%, $n=10$ ) and ob/ob (ob/ob E 0.075\%, $n=10$; ob/ob E 0.15\%, $n=3$ ) mice is shown as mean \pm s.e.m. Sirius Red staining (d, e) in representative lean (WT E 0.15\%) (d) and ob/ob (ob/ob E 0.15\%) (e) after 1 week of high-dose ethionine treatment ( $\times 630$ magnification). Quantification of Sirius red-stained fibrils (f), graphed as mean \pm s.e.m. $\left({ }^{*} P<0.005 \%\right.$ vs WT E $0.15 \% ;{ }^{*} P<0.001 \%$ vs WT E $\left.0.15 \%\right)$.

brief 1-week treatment period, hepatic hydroxyproline content also tripled in ob/ob mice, but remained unchanged in control mice. Thus, the severity of liver fibrosis correlated well with the intensity of Hh pathway activation and accumulation of Hh-responsive cells following injury to mature hepatocytes.

\section{DISCUSSION}

Because chronic oxidative stress activates cell cycle arrest mechanisms in mature hepatocytes, liver regeneration during many types of chronic liver injury requires hepatic progenitor populations. ${ }^{3,45,46}$ The factors mediating the fate of progenitors after oxidative damage to mature hepatocytes remain poorly understood. To investigate this, we employed two well-accepted mouse models of oxidative liver injury, chronic NASH and acute ethionine-induced hepatotoxicity. ${ }^{36,47}$ In both, inhibited proliferation of mature hepatocytes and compensatory expansion of hepatic progenitors have been demonstrated. ${ }^{3,31,37,48,49}$ We hypothesized that $\mathrm{Hh}$ signaling was likely to be involved in the latter process, because Hh ligands orchestrate the development of many tissues, as well as remodeling of damaged epithelia in various adult organs. ${ }^{13,18,19,21,41}$ Although Hh signaling is not known to regulate adult liver regeneration, ${ }^{2}$ evidence that mesendodermal-like cells in fetal livers exhibit Hh pathway activity, ${ }^{11,25,26}$ and that chronic biliary injury induces 
Hedgehog signaling in adult livers, ${ }^{25,26}$ supports this possibility.

The present studies demonstrate a striking dose-response relationship between mature hepatocyte injury and expansion of liver progenitors that produce and respond to $\mathrm{Hh}$ ligands. At baseline, neither lean mice with healthy livers, nor $\mathrm{ob} / \mathrm{ob}$ mice with NASH, exhibited expression of Hh ligands or Hh-target genes in mature hepatocytes. However, $\mathrm{Hh}$ activity was increased in expanded progenitor populations that resided near portal tracts in chronically fatty livers. Ethionine acutely injured mature hepatocytes and increased $\mathrm{Hh}$ signaling and hepatic progenitors in lean mice with previously healthy livers, as well as in ob/ob mice with NASH. All ethionine-elicited responses (ie, mortality, liver injury, $\mathrm{Hh}$ induction, and progenitor accumulation) were greater when the acute injury was superimposed upon chronic liver injury and increased with the dose of ethionine, paralleling the severity of liver injury. These findings extend recent evidence that the Hh pathway is activated by biliary tract injury, ${ }^{25}$ and confirm our hypothesis that diverse types of liver damage in adults, including NASH, promote hepatic accumulation of Hh-responsive cells.

The greatest accumulation of Hh-responsive cells occurred when acute ethionine-induced hepatocyte injury was superimposed upon chronic hepatocyte injury in animals with $\mathrm{NASH}$. These mice also exhibited liver atrophy and increased liver fibrosis. These outcomes correlated with aberrant localization of the Hh-regulated transcription factor, Gli2, in mature-appearing hepatic epithelial cells that concomitantly failed to express detectable levels of HNF-6, a transcription factor that is normally induced during liver epithelial cell differentiation. ${ }^{40,50}$ The appearance of Gli2 in hepatocytic cells was surprising because during development and repair of other tissues, Hh ligand production is most conspicuous in epithelial cells and Hh target gene expression localizes in mesenchymal cells. ${ }^{20,51,52}$ Following even mild acute or chronic injury to hepatocytes, however, we noted that expression of the Hh ligand, Ihh, and the Hh target, Gli2, tended to localize in very close proximity (and possibly colocalize) within bile ductular cells in ethionine-treated mice with NASH. Cells that coexpress epithelial and mesenchymal genes are generally considered to be undergoing epithelial-tomesenchymal (or mesenchymal-to-epithelial) transition. ${ }^{53}$ Mesenchymal-epithelial transitions are a characteristic of mesendodermal progenitors and their immediate progeny. ${ }^{7,8}$ The former are capable of differentiating along various lineages and can generate various types of epithelial cells, ${ }^{7,8}$ including hepatocytes. ${ }^{10}$

The Hh pathway regulates the growth and differentiation of mesendodermal cells. ${ }^{13}$ Recently, mesendodermal-like cells that produce and respond to Hh ligands were isolated from human fetal livers. ${ }^{11}$ The present findings suggest that some of these cells, or their immediate progeny, persist in adult livers. Consistent with current understanding of the progenitor compartment in adult livers, ${ }^{2}$ such cells localize near
PT and increase in number when the death rate of mature hepatocytes exceeds the proliferative capacity of the residual surviving hepatocytes. In the present studies, superimposing acute ethionine-induced hepatotoxicity upon chronic NASHrelated liver injury elicited the most severe hepatic parenchymal injury and resulted in the greatest PT accumulation of Gli2 $(+)$ stromal cells and bile ductular cell populations that express Ihh and Gli2. Also, in contrast to mature-appearing hepatocytes in healthy or less-severely injured livers, many mature-appearing hepatocytes in livers with acute-onchronic liver injury expressed the mesenchymal gene product, Gli2, ${ }^{13}$ but lacked obvious expression of HNF-6, a liver epithelial transcription factor. ${ }^{40,50}$ Implications for the derivation of mature hepatocytes, bile ductular cells or PT mesenchymal cells remain uncertain, and merit further research.

Since matrix production is a well-accepted characteristic of mesenchymal cells, ${ }^{53}$ Gli2 expression in bile ductular and hepatocytic cells of severely injured livers suggests a novel mechanism for liver fibrosis. Namely, hepatic epithelial cells may acquire certain features of mesenchymal cells and contribute to liver fibrosis during severe parenchymal liver injury. This concept is supported by the increased collagen mRNA and Sirius red-stained fibrils in ethionine-treated ob/ ob mice. Activation of hepatic stellate cells to collagen-producing myofibroblasts is severely inhibited by leptin deficiency, ${ }^{43,44}$ implicating other cell types in the ob/ob fibrogenic response to ethionine. Similar logic suggests that the increased levels of collagen gene expression that we noted in ob/ob mice at baseline were not likely due to accumulation of myofibroblastic stellate cells. Recently, other groups demonstrated that fibrosis correlated with the ductular response to oxidative liver injury in mice with normal leptin genes, ${ }^{54}$ and in patients with various types of chronic liver disease. ${ }^{46,55,56}$ Hence, participation of epithelial-mesenchymal transitions in hepatic fibrogenesis may not be limited to leptin-deficient mice.

Our data suggest that adult livers respond to some types of damage by evoking mechanisms that are sometimes induced in other injured adult organs. Under pathologic conditions, for example, adult renal tubular epithelial cells undergo epithelial to mesenchymal transition, and this phenotypic conversion is believed to play a critical role in renal fibrosis. ${ }^{53}$ Evidence suggests that the subpopulations of adult renal tubular cells that are capable of epithelial-to-mesenchymal transition are progeny of mesenchymal cells that migrated into developing kidney tubules and underwent mesenchymal-to-epithelial transition during embryogenesis. ${ }^{57}$ This suggests that re-activation of mechanisms, including $\mathrm{Hh}$ signaling, that regulate such epithelial-mesenchymal interactions during renal development, might modulate fibroproliferative responses in adult kidneys. A similar concept is being investigated in pulmonary fibrosis. ${ }^{58}$ The present study demonstrates that fatty liver damage activates the Hh pathway and provides evidence supporting epithelialmesenchymal transitions in adult liver cells. These findings 
identify novel mechanisms for liver regeneration/repair and have potentially important diagnostic and therapeutic implications for patients with NASH.

\section{ACKNOWLEDGEMENT}

This work was supported by National Institutes of Health Grants 5RO1AA010154-11 (Anna Mae Diehl) and 5RO1-DK053792-06 (Anna Mae Diehl).

1. Feldstein $A E$, Gores GJ. Apoptosis in alcoholic and nonalcoholic steatohepatitis. Front Biosci 2005;10:3093-3099.

2. Fausto N, Campbell JS. The role of hepatocytes and oval cells in liver regeneration and repopulation. Mech Dev 2003;120:117-130.

3. Roskams T, Yang SQ, Koteish A, et al. Oxidative stress and oval cell accumulation in mice and humans with alcoholic and nonalcoholic fatty liver disease. Am J Pathol 2003;163:1301-1311.

4. Yang $\mathrm{SQ}$, Lin $\mathrm{HZ}$, Mandal $\mathrm{AK}$, et al. Disrupted signaling and inhibited regeneration in obese mice with fatty livers: implications for nonalcoholic fatty liver disease pathophysiology. Hepatology 2001;34(Part 1):694-706.

5. Kuhlmann WD, Peschke P. Hepatic progenitor cells, stem cells, and AFP expression in models of liver injury. Int J Exp Pathol 2006;87: 343-359.

6. Yovchev Ml, Grozdanov PN, Joseph B, et al. Novel hepatic progenitor cell surface markers in the adult rat liver. Hepatology 2007:45:139-149.

7. McLean $A B, D^{\prime} A m o u r ~ K A$, Jones $K L$, et al. Activin a efficiently specifies definitive endoderm from human embryonic stem cells only when phosphatidylinositol 3-kinase signaling is suppressed. Stem Cells 2007:25:29-38.

8. Tada S, Era T, Furusawa $C$, et al. Characterization of mesendoderm: a diverging point of the definitive endoderm and mesoderm in embryonic stem cell differentiation culture. Development 2005;132:4363-4374.

9. Fletcher $G$, Jones $G E$, Patient $R$, et al. A role for GATA factors in Xenopus gastrulation movements. Mech Dev 2006;123:730-745.

10. Dan YY, Riehle KJ, Lazaro C, et al. Isolation of multipotent progenitor cells from human fetal liver capable of differentiating into liver and mesenchymal lineages. Proc Natl Acad Sci USA 2006;103:9912-9917.

11. Sicklick JK, Li YX, Melhem A, et al. Hedgehog signaling maintains resident hepatic progenitors throughout life. Am J Physiol Gastrointest Liver Physiol 2006;290:G859-G870.

12. Hooper JE, Scott MP. Communicating with Hedgehogs. Nat Rev Mol Cell Biol 2005;6:306-317.

13. Dwyer JR, Sever N, Carlson M, et al. Oxysterols are novel activators of the hedgehog signaling pathway in pluripotent mesenchymal cells. J Biol Chem 2007;282:8959-8968.

14. Kimelman D, Griffin KJ. Vertebrate mesendoderm induction and patterning. Curr Opin Genet Dev 2000;10:350-356.

15. Li X, Deng W, Lobo-Ruppert SM, et al. Gli1 acts through snail and $\mathrm{E}$-cadherin to promote nuclear signaling by beta-catenin. Oncogene 2007;26:4489-4498.

16. Marion NW, Mao JJ. Mesenchymal stem cells and tissue engineering. Methods Enzymol 2006;420:339-361.

17. Zipori D. Mesenchymal stem cells: harnessing cell plasticity to tissue and organ repair. Blood Cells Mol Dis 2004;33:211-215.

18. Adolphe $C$, Wainwright B. Pathways to improving skin regeneration Expert Rev Mol Med 2005;7:1-14.

19. Kusano KF, Pola R, Murayama T, et al. Sonic hedgehog myocardial gene therapy: tissue repair through transient reconstitution of embryonic signaling. Nat Med 2005;11:1197-1204.

20. Warburton $D$, Tefft $D$, Mailleux $A$, et al. Do lung remodeling, repair, and regeneration recapitulate respiratory ontogeny? Am J Respir Crit Care Med 2001;164(Part 2):S59-S62.

21. Williams JA. Hedgehog and spinal cord injury. Expert Opin Ther Targets 2005;9:1137-1145.

22. Berman DM, Karhadkar SS, Maitra A, et al. Widespread requirement for Hedgehog ligand stimulation in growth of digestive tract tumours. Nature 2003;425:846-851.

23. Sicklick JK, Li YX, Jayaraman A, et al. Dysregulation of the Hedgehog pathway in human hepatocarcinogenesis. Carcinogenesis 2006;27:748-757.
24. Toftgard R. Hedgehog signalling in cancer. Cell Mol Life Sci 2000;57:1720-1731.

25. Omenetti A, Yang L, Li YX, et al. Hedgehog-mediated mesenchymalepithelial interactions modulate hepatic response to bile duct ligation. Lab Invest 2007:87:499-514.

26. Jung Y, McCall SJ, Li YX, et al. Bile ductules and stromal cells express hedgehog ligands and/or hedgehog target genes in primary biliary cirrhosis. Hepatology 2007;45:1091-1096.

27. Bataller R, Brenner DA. Liver fibrosis. J Clin Invest 2005;115:209-218.

28. Ramadori G, Saile B. Portal tract fibrogenesis in the liver. Lab Invest 2004:84:153-159.

29. McCullough AJ. The clinical features, diagnosis and natural history of nonalcoholic fatty liver disease. Clin Liver Dis 2004;8:521-533, viii.

30. $\mathrm{Xu} \mathrm{A}$, Wang $\mathrm{Y}$, Keshaw $\mathrm{H}$, et al. The fat-derived hormone adiponectin alleviates alcoholic and nonalcoholic fatty liver diseases in mice. J Clin Invest 2003;112:91-100.

31. Yang $\mathrm{S}$, Koteish $\mathrm{A}$, Lin $\mathrm{H}$, et al. Oval cells compensate for damage and replicative senescence of mature hepatocytes in mice with fatty liver disease. Hepatology 2004;39:403-411.

32. Yang L, Chan CC, Kwon OS, et al. Regulation of peroxisome proliferator-activated receptor-gamma in liver fibrosis. Am J Physiol Gastrointest Liver Physiol 2006;291:G902-G911.

33. Yang L, Magness ST, Bataller R, et al. NF-kappaB activation in Kupffer cells after partial hepatectomy. Am J Physiol Gastrointest Liver Physiol 2005;289:G530-G538.

34. Choi SS, Sicklick JK, Ma Q, et al. Sustained activation of Rac1 in hepatic stellate cells promotes liver injury and fibrosis in mice. Hepatology 2006; $44: 1267-1277$.

35. Padma $\mathrm{P}$, Setty $\mathrm{OH}$. Protective effect of vitamin $\mathrm{E}$ against ethionine toxicity. Biochem Mol Biol Int 1997;41:785-795.

36. Nanji AA. Animal models of nonalcoholic fatty liver disease and steatohepatitis. Clin Liver Dis 2004;8:559-574 ix.

37. Akhurst B, Croager EJ, Farley-Roche CA, et al. A modified cholinedeficient, ethionine-supplemented diet protocol effectively induces oval cells in mouse liver. Hepatology 2001;34:519-522.

38. Tee LB, Kirilak Y, Huang WH, et al. Differentiation of oval cells into ductlike cells in preneoplastic liver of rats placed on a choline-deficient diet supplemented with ethionine. Carcinogenesis 1994;15:2747-2756.

39. Akhurst B, Matthews V, Husk K, et al. Differential lymphotoxin-beta and interferon gamma signaling during mouse liver regeneration induced by chronic and acute injury. Hepatology 2005;41:327-335.

40. Rausa F, Samadani U, Ye H, et al. The cut-homeodomain transcriptional activator HNF-6 is coexpressed with its target gene HNF-3 beta in the developing murine liver and pancreas. Dev Biol 1997;192:228-246.

41. Katoh $Y$, Katoh M. Hedgehog signaling pathway and gastrointestinal stem cell signaling network (review). Int J Mol Med 2006;18: 1019-1023.

42. Kouros-Mehr H, Werb Z. Candidate regulators of mammary branching morphogenesis identified by genome-wide transcript analysis. Dev Dyn 2006;235:3404-3412.

43. Leclercq IA, Farrell GC, Schriemer R, et al. Leptin is essential for the hepatic fibrogenic response to chronic liver injury. J Hepatol 2002;37:206-213.

44. Saxena NK, Ikeda K, Rockey DC, et al. Leptin in hepatic fibrosis: evidence for increased collagen production in stellate cells and lean littermates of ob/ob mice. Hepatology 2002;35:762-771.

45. Crary GS, Albrecht JH. Expression of cyclin-dependent kinase inhibitor p21 in human liver. Hepatology 1998;28:738-743.

46. Marshall A, Rushbrook S, Davies SE, et al. Relation between hepatocyte G1 arrest, impaired hepatic regeneration, and fibrosis in chronic hepatitis C virus infection. Gastroenterology 2005:128:33-42.

47. Chitturi S, Farrell GC. Etiopathogenesis of nonalcoholic steatohepatitis. Semin Liver Dis 2001:21:27-41.

48. Braun L, Mikumo R, Fausto N. Production of hepatocellular carcinoma by oval cells: cell cycle expression of c-myc and p53 at different stages of oval cell transformation. Cancer Res 1989;49:1554-1561.

49. Tatematsu $M$, Hasegawa $R$, Imaida $K$, et al. Survey of various chemicals for initiating and promoting activities in a short-term in vivo system based on generation of hyperplastic liver nodules in rats. Carcinogenesis 1983;4:381-386.

50. Clotman F, Lannoy VJ, Reber M, et al. The onecut transcription factor HNF6 is required for normal development of the biliary tract. Development 2002;129:1819-1828. 
51. Boras-Granic K, Chang H, Grosschedl R, et al. Lef1 is required for the transition of Wnt signaling from mesenchymal to epithelial cells in the mouse embryonic mammary gland. Dev Biol 2006;295:219-231.

52. Gritli-Linde A, Hallberg K, Harfe BD, et al. Abnormal hair development and apparent follicular transformation to mammary gland in the absence of hedgehog signaling. Dev Cell 2007;12:99-112.

53. Kalluri R, Neilson EG. Epithelial-mesenchymal transition and its implications for fibrosis. J Clin Invest 2003;112:1776-1784.

54. Knight B, Akhurst B, Matthews VB, et al. Attenuated liver progenitor (oval) cell and fibrogenic responses to the choline deficient, ethionine supplemented diet in the BALB/c inbred strain of mice. J Hepatol 2007:46:134-141.
55. Clouston $A D$, Powell $E E$, Walsh $\mathrm{MJ}$, et al. Fibrosis correlates with a ductular reaction in hepatitis $C$ : roles of impaired replication, progenitor cells and steatosis. Hepatology 2005;41:809-818.

56. Ray MB, Mendenhall CL, French SW, et al. Bile duct changes in alcoholic liver disease. The Veterans Administration Cooperative Study Group. Liver 1993;13:36-45.

57. Denholm B, Sudarsan V, Pasalodos-Sanchez S, et al. Dual origin of the renal tubules in Drosophila: mesodermal cells integrate and polarize to establish secretory function. Curr Biol 2003;13: 1052-1057.

58. Willis $B C$, duBois RM, Borok Z. Epithelial origin of myofibroblasts during fibrosis in the lung. Proc Am Thorac Soc 2006;3:377-382. 